\title{
Resource sharing controls gene expression bursting
}

Patrick M. Caveney ${ }^{1,2}$, S. Elizabeth Norred ${ }^{1,2}$, Charles W. Chin ${ }^{1,2}$, Jonathan B. Boreyko $0^{1,2,3}$, Brandon S. Razooky ${ }^{2,4}$, Scott T. Retterer ${ }^{1,2,5}$, C. Patrick Collier ${ }^{2}$, Michael L. Simpson ${ }^{\Uparrow 1,2,6}$

${ }^{1}$ Bredesen Center, University of Tennessee, Knoxville, Tennessee 37996-2010, United States

${ }^{2}$ Center for Nanophase Materials Sciences, Oak Ridge National Laboratory Bethel Valley Road, Oak Ridge, Tennessee 37831, United States

${ }^{3}$ Department of Biomedical Engineering and Mechanics, Virginia Tech, Blacksburg, Virginia 24061, United States

${ }^{4}$ Laboratory of Immune Cell Epigenetics and Signaling, The Rockefeller University, New York, New York 10065, United States

${ }^{5}$ Biosciences Division, Oak Ridge National Laboratory, Oak Ridge, Tennessee 37831, United States

${ }^{6}$ Joint Institute for Biological Sciences, University of Tennessee, Knoxville and Oak Ridge National Laboratory, Bethel Valley Road, Oak Ridge, Tennessee 37831, United States

Correspondence should be addressed to MLS (SimpsonML1@ornl.gov)

This manuscript has been authored by UT-Battelle, LLC under Contract No. DE-AC05-00OR22725 with the U.S. Department of Energy. The United States Government retains and the publisher, by accepting the article for publication, acknowledges that the United States Government retains a non-exclusive, paid-up, irrevocable, world-wide license to publish or reproduce the published form of this manuscript, or allow others to do so, for United States Government purposes. The Department of Energy will provide public access to these results of federally sponsored research in accordance with the DOE Public Access Plan(http://energy.gov/downloads/doe-public-access-plan). 


\section{Supplemental Information}

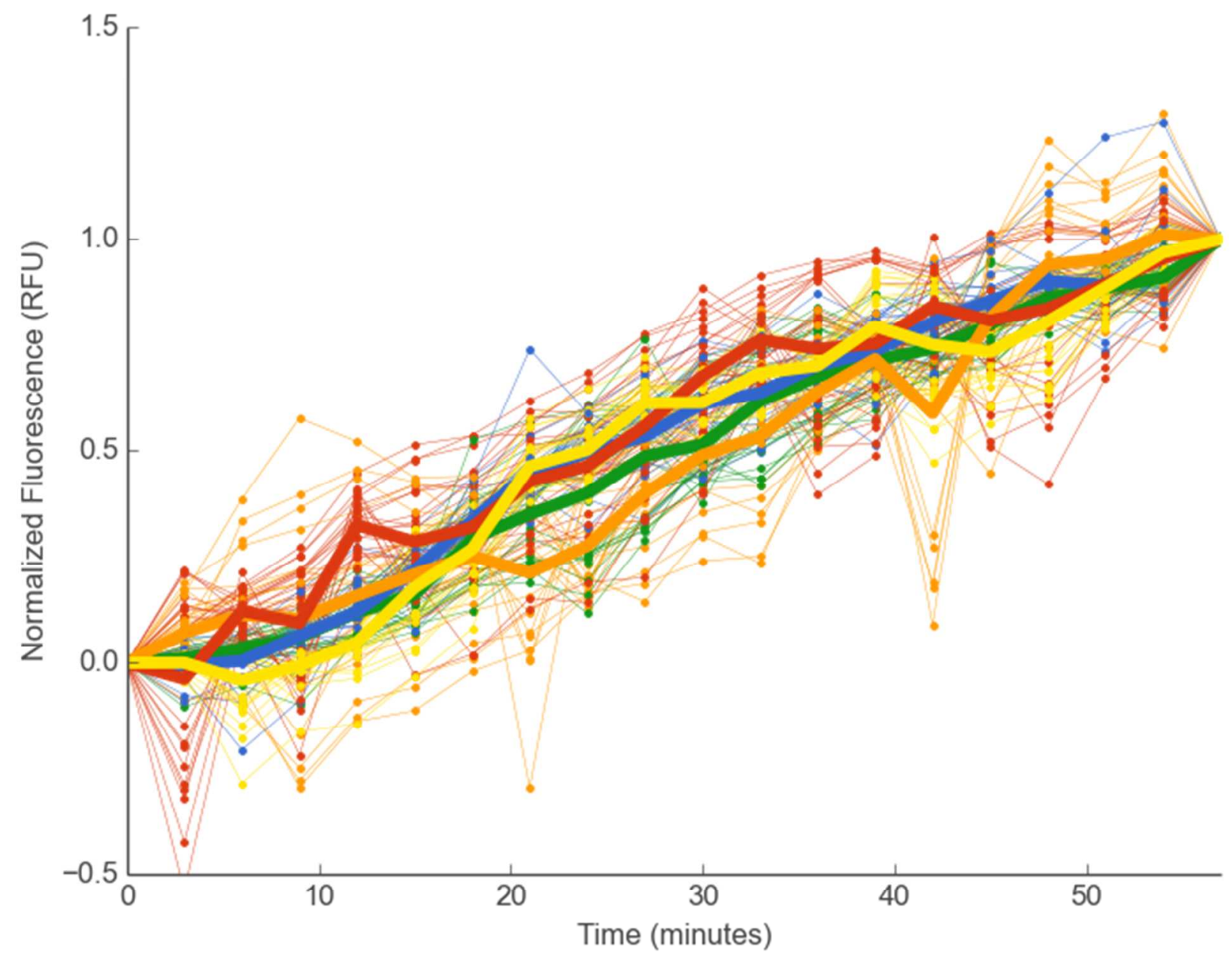

Supplemental Figure 1 Comparison of transient behavior between different experimental days. Each color shows the average normalized fluorescence transient on a particular experimental day. Thin lines are individual chambers while thick lines are averages of chambers of all sizes acquired in the same experimental session. Each experiment had a similar $50 \%$ rise time ( $\sim 25$ minutes) and GFP production rate. 


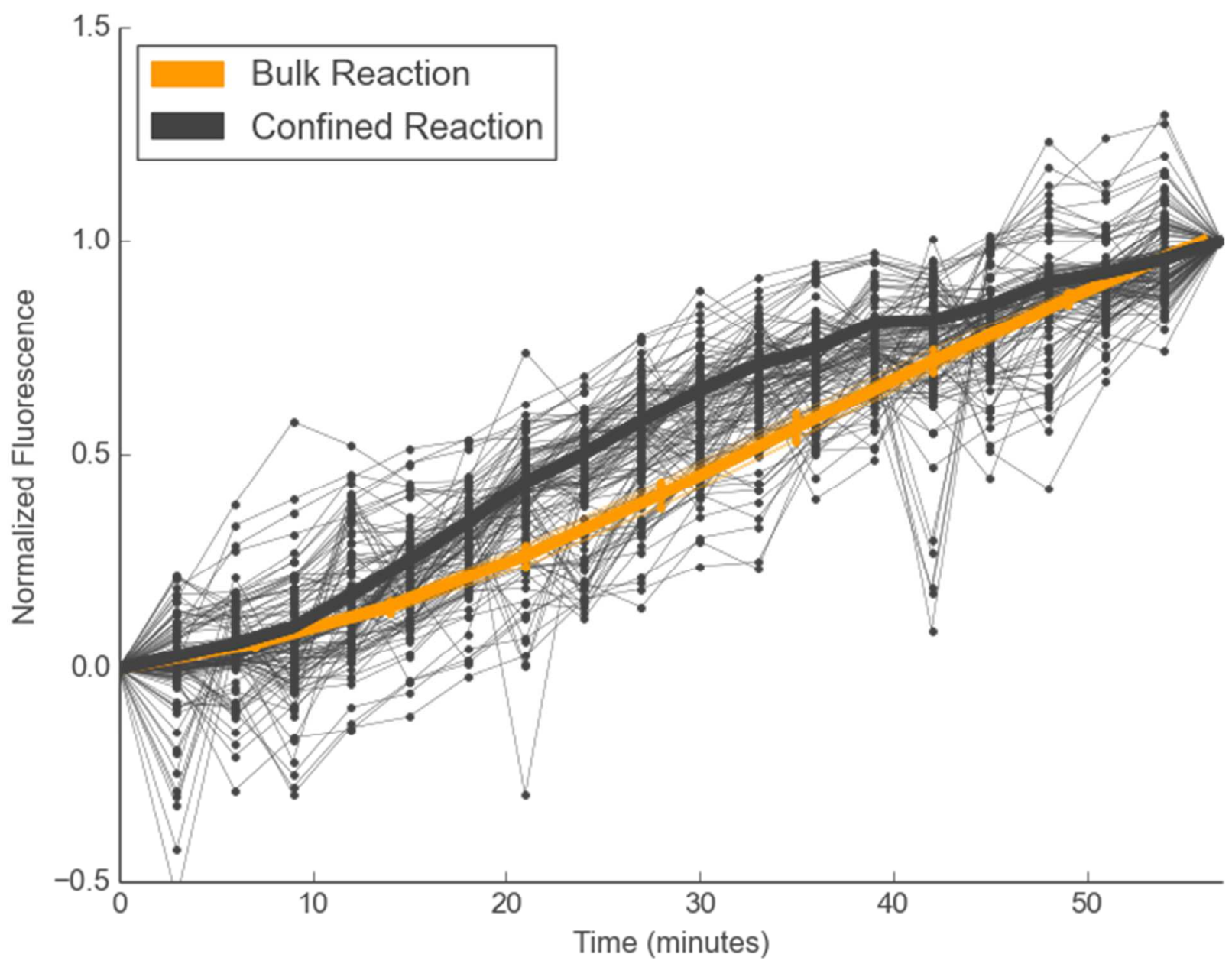

Supplementary Figure $\mathbf{2}$ Comparison of fluorescence transients in unconfined (i.e. bulk) and confined chambers. Bulk reactions were run in a BioTek Synergy 2 plate reader. 25 $\mu \mathrm{L}$, commercial, raw extract, cell-free protein synthesis (CFPS) reactions were mixed with 500ng of Enhanced Green Fluorescent Protein (EGFP) coding pET3a plasmid, and pipetted into a 384 well plate. Reactions were covered with $10 \mu \mathrm{L}$ of mineral oil to prevent evaporation. Excitation was at $485 \mathrm{~nm}$ and fluorescence was measured at 528 nm every 7 minutes for 1 hour. 


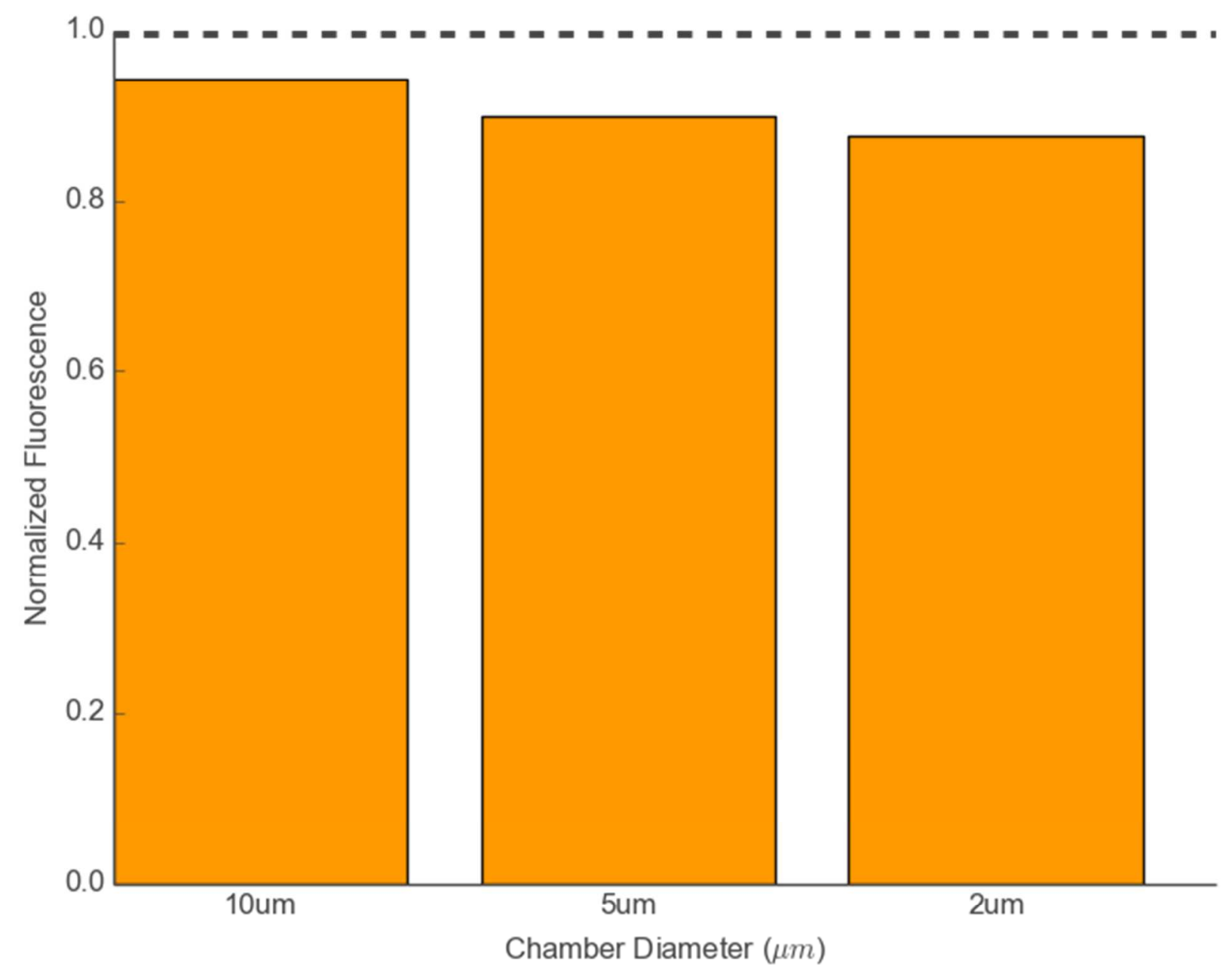

Supplementary Figure 3 Photobleaching during imaging. To measure the effect of photobleaching occurring during experiments we ran a standard experiment for one hour with the aperture narrowed to only the 9 chambers in the field of view. At the end of the experiment we compared the intensity of the 9 chambers in the field of view to the intensity of 9 chambers of the same size, on the same chip, during the same reaction that had not been exposed. Fluorescence intensity was only slightly reduced (by about $10 \%$, dashed gray line vs. bars) and was reduced by similar amounts for all chamber sizes. 


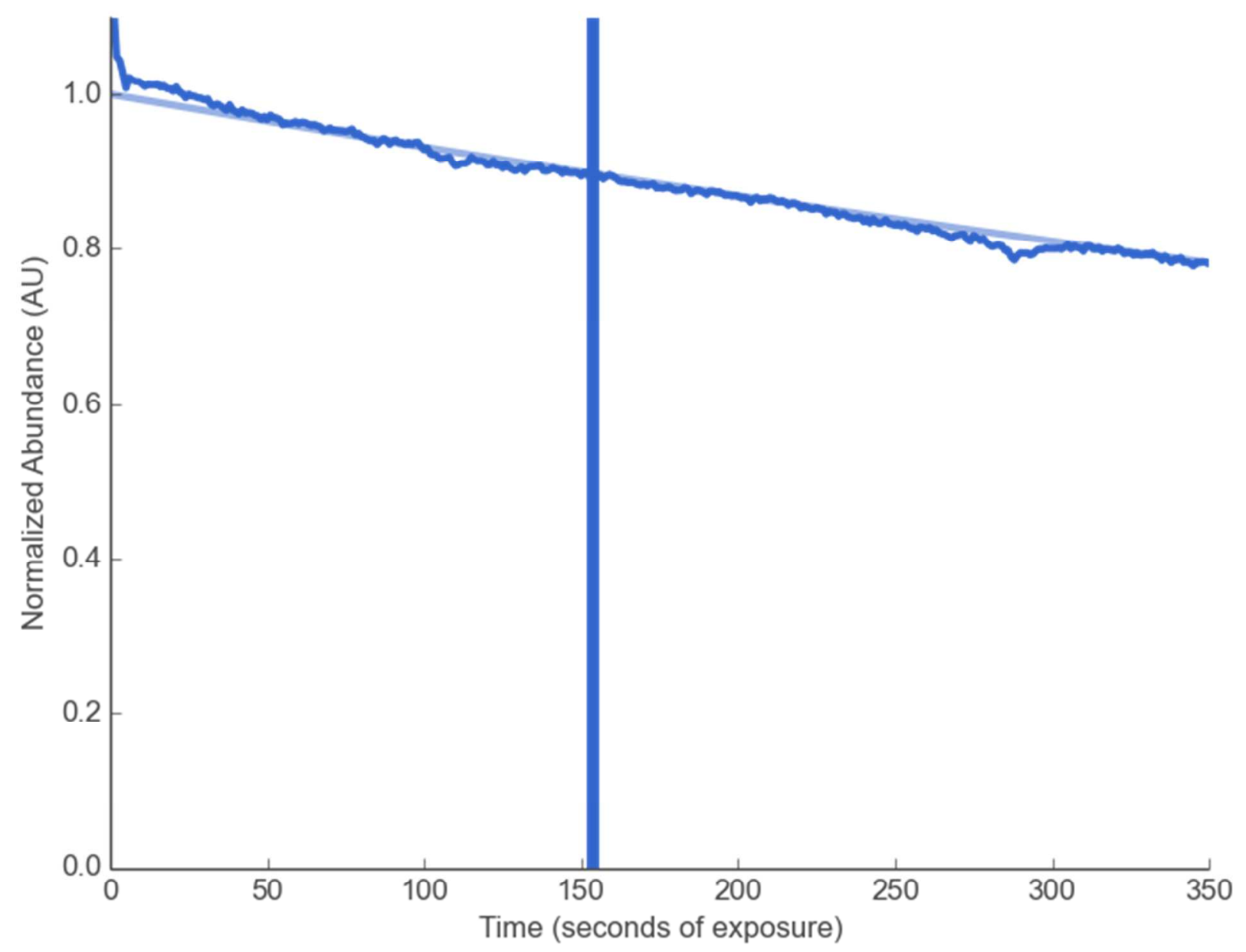

Supplementary Figure 4 Rate of photobleaching. To measure the photobleaching rate in the system we imaged confined reactions in a standard experiment for one hour. We then continuously exposed the reaction to the light source (Nikon Intensilight C-HGFI) for 10 minutes while recording an image every second. The average intensity of 9 chambers was recorded for each time point and plotted against exposure time. Fluorescent intensity was normalized to the initial value and fit with an exponential decay curve. The photobleaching half-life was measured to be 987.47 seconds. The vertical line corresponds to the measured amount of photobleaching during a one-hour experiment, 153 seconds of exposure and a $10 \%$ reduction in fluorescence compared to chambers not imaged. 


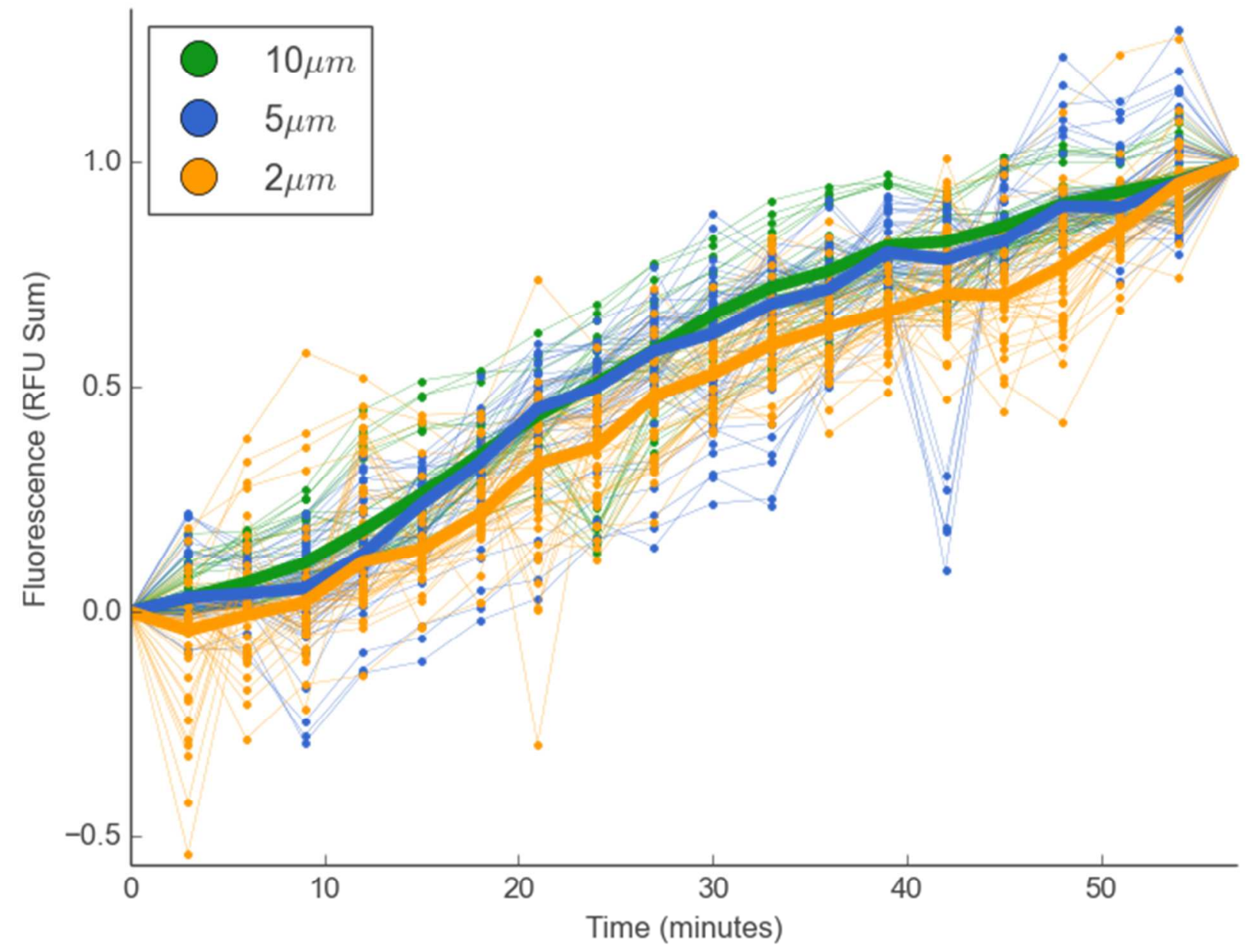

Supplemental Figure 5 Effect of chamber size on fluorescence transient. Normalized fluorescent traces show the transients of different chamber sizes exhibit very similar behavior. Thick lines represent the average behavior of all chambers of a given size, while the thin lines depict individual chamber behavior. Both the average transient behavior and the distributions of the individual behaviors show little variation across the different chamber sizes. 


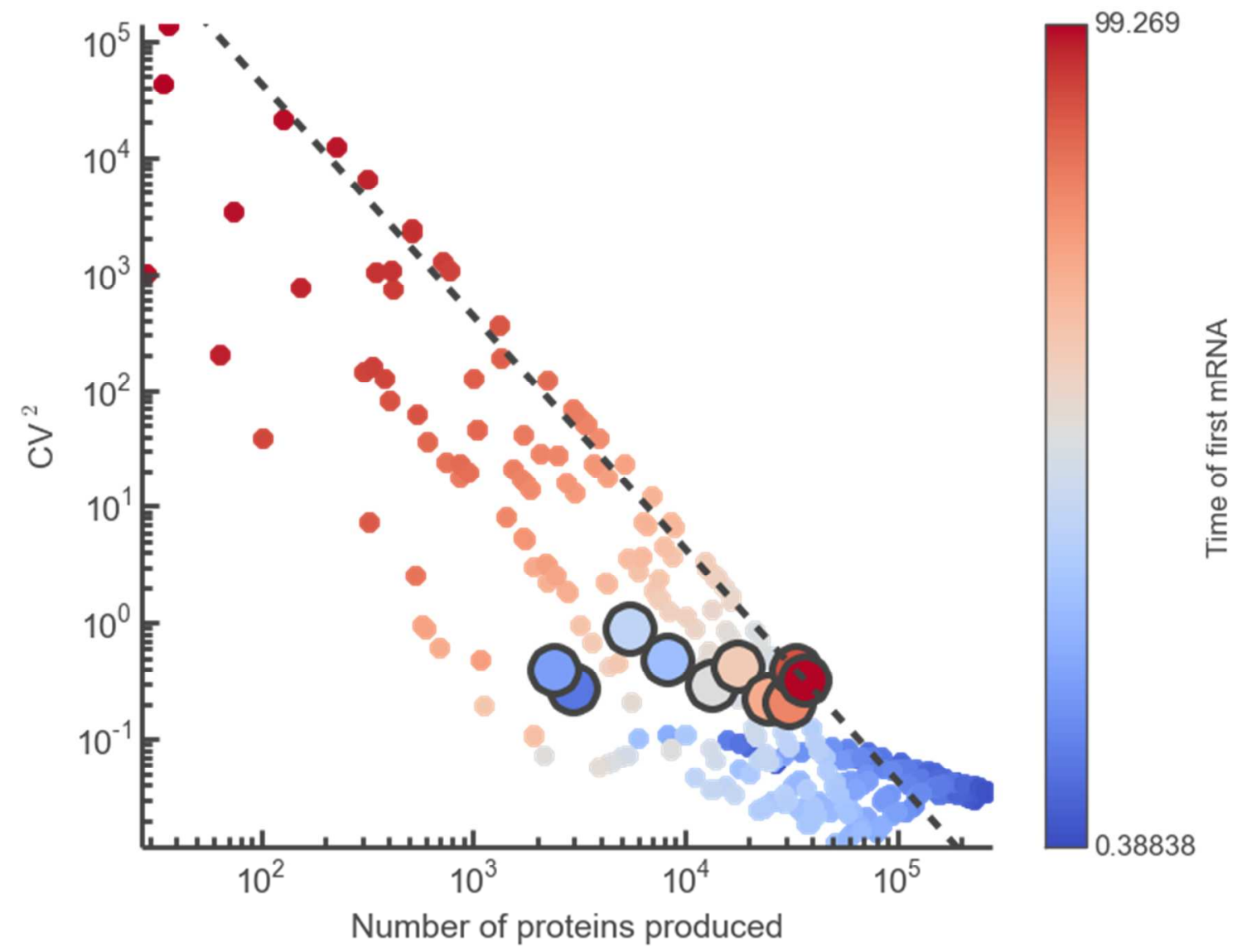

Supplementary Figure 6 Noise plot of simulation data colored by the time the first mRNA was made. Centroids are colored by reaction size (blue 15 genes; red 150 genes). The dashed gray line has the form a/abundance ${ }^{2}$. The timing of mRNA production heavily influences both the amount of protein produced and the noise. 


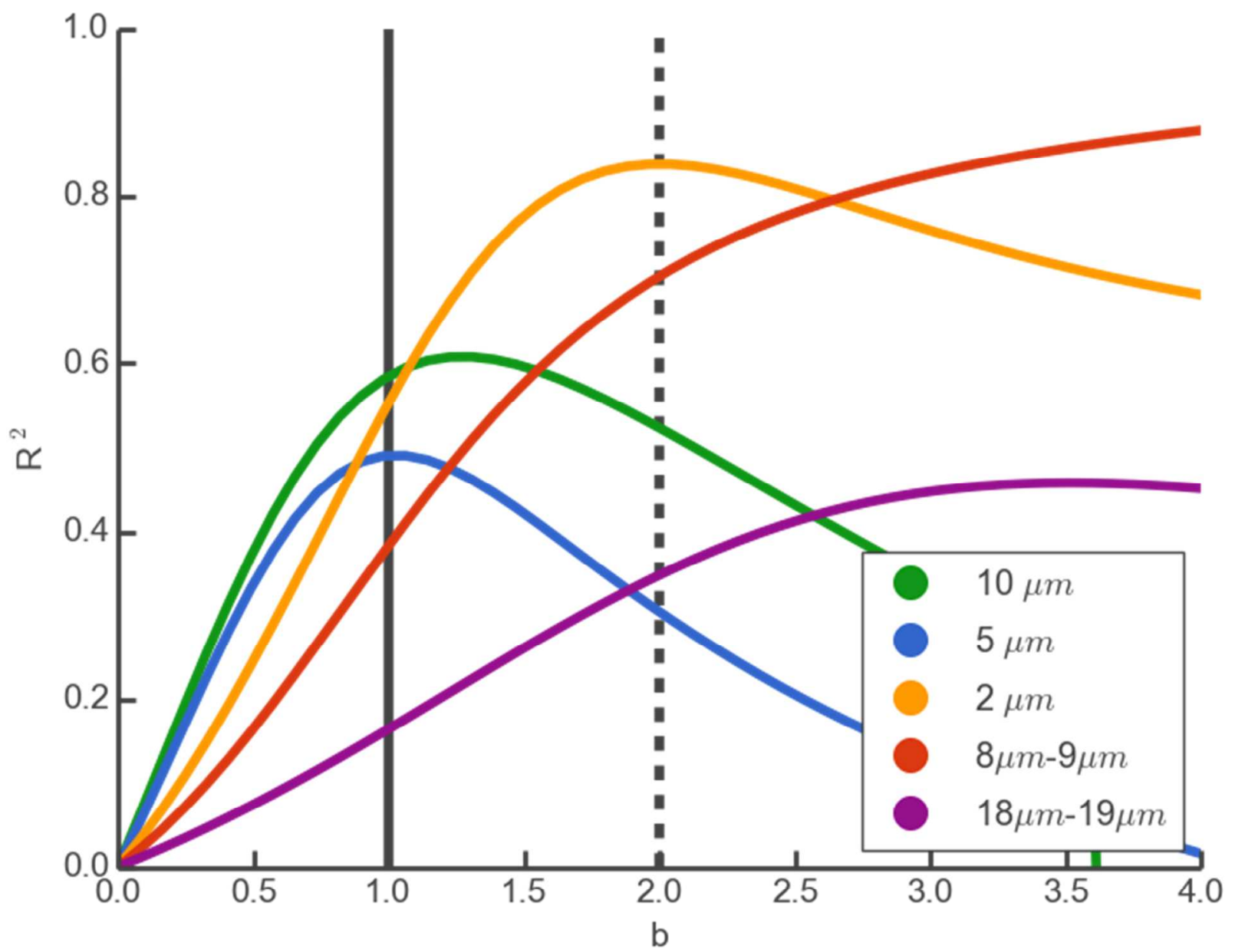

Supplementary Figure $7 \mathrm{R}^{2}$ for varying power law fits to experimental data. Chamber and vesicle noise plot data (Fig. $3 a$ and $3 c$ ) were fit with equations of the form $a^{*}$ abundance ${ }^{-b}$. Values of $b$ ranged between 0.0 and 4.0. For each value of $b$, the value of a was found by nonlinear least squared fitting. $R^{2}$ was measured and plotted for each value of $b$. Values of $b$ between 1.0 (solid line) and 2.0 (dashed line) show good fits to the experimental data in both chambers and vesicles. 